EXEMPLARIa Classica

Journal of Classical Philology

23, 2019, 109-122 • ISSN 1699-3225

http://dx.doi.org/10.33776/ec.v23i0.3668

\title{
COLACIÓN DE LAS EPÍSTOLAS DE HORACIO EN EL MS. ROTENSIS 7 (ARCHIVO CAPITULAR DE LÉRIDA)*
}

\author{
Víctor SABATÉ VidAL \\ Universidad de Barcelona \\ vsabatev@gmail.com \\ ORCID: 0000-0003-1699-8111
}

\section{SUMMARY}

This paper offers a collation of a twelfthcentury manuscript of Horace's Epistles, namely the MS Rotensis 7 of the Chapter Archive in Lleida, which used to be conserved at the cathedral of Roda d'Isàvena, where it appears to have been copied. The paper also includes the physical description of the manuscript and, while the stemma codicum of the works of Horace still awaits close study, some possible connections with other codices are pointed out.

KEYWORDS

Latin textual criticism; Horace; Epistles; manuscript; collation; Roda d'Isàvena.

\section{RESUMEN}

Se ofrece la colación del texto de las Epístolas de Horacio contenido en el manuscrito Rotensis 7, códice del siglo XII que se conserva en el Archivo Capitular de Lérida pero que procede de los fondos de la catedral de Roda de Isábena, donde parece que fue copiado. Se incluye también la descripción física del manuscrito $\mathrm{y}$, a pesar de que el establecimiento del estema de la transmisión de Horacio está pendiente de un estudio completo de sus testimonios, se señalan algunas posibles conexiones entre el nuestro y otros códices.

Palabras Clave

Crítica textual latina; Horacio; Epistolas; manuscrito; colación; Roda de Isábena.

Vaya en primer lugar mi agradecimiento a Joan J. Busqueta, quien me dio a conocer este manuscrito hace ya algunos años y me facilitó los materiales necesarios para emprender su estudio. Los principales responsables de que la empresa haya llegado a buen puerto han sido Juan Antonio Estévez Sola y Antonio Ramírez de Verger, a quienes agradezco asimismo sus orientaciones, insistencia y paciencia. El texto debe mucho a las atentas lecturas y correcciones de Julia Aguilar y Dániel Kiss, que lo han salvado de innumerables errores e imprecisiones. Por último, he de dar las gracias al Archivo Capitular de Lérida y, en particular, a Anna Carles por las atenciones recibidas durante mi visita a su sede en julio y octubre de 2019. El trabajo ha sido realizado merced a una ayuda del Ministerio de Educación para la Formación de Profesorado Universitario (FPU2014/00944), en el seno del proyecto FFI2015-68571-P y del GRC LITTERA de la Universidad de Barcelona (2017SGR241).

Fecha de recepción: 25/07/2019

Fecha de aceptación y versión definitiva: 16/10/2019 
Àngel Mestres Tibau daba noticia, en 1949, de la existencia de un códice horaciano en Lérida, y ofrecía un estudio preliminar de este testimonio manuscrito, sin duda el más importante de los custodiados en el Archivo Capitular de aquella sede ${ }^{1}$. El documento ha sido posteriormente recogido en varios catálogos, entre los que cabe destacar el de Lisardo Rubio ${ }^{2}$, pero ninguno de los editores de Horacio le ha prestado la menor atención, hecho que motiva el presente estudio. Es particularmente llamativa su ausencia en las ediciones de Fernando Navarro Antolín (Alma Mater), cuyo aparato crítico atesora las lecturas de numerosos códices españoles hasta el momento inéditos ${ }^{3}$, y de Isidor Ribas (Fundació Bernat Metge), que en cambio sí llevó a cabo la primera colación de otro manuscrito conservado en Cataluña, el Dertusensis 100 (Archivo Capitular de Tortosa) ${ }^{4}$.

\section{Descripción del ms. Rotensis 7 DEl Archivo CaPitular de LÉRIDA}

El ms. Rotensis 7 (en adelante $L$ ) ${ }^{5}$ es un códice de pergamino de unos $185 \mathrm{x}$ $120 \mathrm{~mm}$, con cubierta y sobrecubierta $(215 \times 147 \mathrm{~mm})$ también en pergamino. Consta de 17 folios en cuarto distribuidos en dos cuadernos (ff. 1-8 y 9-17). El segundo cuaderno contaba inicialmente con 10 folios, pero la primera hoja del segundo bifolio fue cortada ya antes de la copia del texto, quedando así desparejado el f. 16, que es sensiblemente más estrecho que el resto. No se observan reclamos ni numeración de cuadernos. El manuscrito presenta líneas de pautado a punta seca y numeración arábiga moderna a lápiz en la esquina superior derecha del recto de los folios. Su estado de conservación es bueno:

1 À. Mestres Tibau, "Un manuscrito horaciano en Lérida”, Ilerda 12-13, 1949, 73-81.

${ }^{2}$ L. Rubio Fernández, Catálogo de los manuscritos clásicos latinos existentes en España, Madrid 1984, nº 350. Véase también A. Cordoliani, "Inventaire des manuscrits de comput ecclésiastique conservés dans les bibliothèques de Catalogne (avec notes sur les autres manuscrits de ces bibliothèques)", Hispania Sacra 4, 1951, 359-84, esp. 378; Y.-F. Riou, "Codicologie et notation neumatique", Cahiers de civilisation médiévale 33, 1990, 255-80, esp. 267; F. Castillón Cortada, "Catálogo del archivo de la Catedral de Lleida. Fondos de Roda de Isábena”, Aragonia Sacra 9, 1994, 133-92, esp. 137, nº 2; R. Swanson Hernández, Tradicions $i$ transmissions iconogràfiques dels manuscrits de la Ribagorça entre els segles X-XII, 2 vols., Barcelona 2016, II, 27-36, nº 2 [<hdl.handle.net/10803/401465>].

${ }^{3}$ F. Navarro Antolín, Quinto Horacio Flaco. Epistolas. Arte poética, Madrid 2002.

${ }^{4}$ I. Ribas Bassa, L. Riber i Campins, Q. Horaci Flac. Sàtires i epístoles, Barcelona 1927.

${ }^{5}$ ACL, RC_0004 (antiguo R-07). Pace Mestres, "Un manuscrito", 77, que opta con excesiva prudencia por la denominación de Ilerdensis, me decanto aquí por conservar la tradicional de Rotensis 7, dada la más que segura pertenencia del códice a los fondos de Roda de Isábena (infra, § 2). Para la sigla sí me baso en el nombre de la ciudad que lo acoge hoy, puesto que la letra $L$ no aparece empleada ni en la edición que se toma como referencia para la colación (D. R. Shackleton Bailey, Q. Horatius Flaccus. Opera, Berlín-Nueva York $2008^{4}$ [2001]) ni en la de Navarro Antolín, Epistolas, mientras que, desde la editio maior de O. Keller y A. Holder, $Q$. Horati Flacci Opera, 2 vols., Leipzig 1864-9, $R$ suele hacer referencia al Vaticanus Reg. lat. 1703. 
hay manchas de humedad en unas pocas hojas (ff. $2^{\mathrm{r}}, 3^{\mathrm{r}}$ y $\left.3^{\mathrm{v}}\right)$, y la mayor parte de los agujeros y recortes que se observan son anteriores al proceso de copia (ff. $2,4,12$ y 14). La única pérdida material posterior se encuentra en la parte inferior del f. 14 y ha comportado la desaparición del comienzo de Hor. epist. 2.1.161-165 (f. 14r) y el final de 2.1.207-210 (f. 14 v).

La mayor parte del códice alberga, en efecto, una copia completa de los dos libros de las Epístolas de Horacio (ff. 1-15 ${ }^{\mathrm{v}}$ y $15^{\mathrm{v}}-17^{\mathrm{r}}$ ), producto del trabajo de un único copista. En la última página (f. $17^{\mathrm{v}}$ ) se aprecian dos manos: una escribe las primeras 22 líneas y media, siendo la misma que ha copiado el poema horaciano en los folios anteriores, y la otra termina la línea empezada y se encarga de las 22 siguientes. La paleografía es muy similar: se trata en ambos casos de una minúscula carolina de trazo anguloso (s. XII), casi de transición, lo que explica que Mestres definiera la letra como gótica ${ }^{6}$. Los copistas se diferencian por el tamaño de las letras (el módulo de las del segundo es ligeramente menor), el color de la tinta (el primer copista utiliza una tonalidad de marrón algo más clara) y pequeños detalles en la forma de parte de las abreviaturas empleadas.

No hay acuerdo en cuanto al contenido de esta última página. Según Mestres "puede leerse una breve esquematización de las Metamorfosis de Ovidio y una serie de notas gramaticales"7. Rebecca Swanson va más allá e identifica partes del comentario de Arnulfo de Orleans a las Metamorfosis de Ovidio, compuesto en torno a 1175: el primer escriba habría copiado un breve resumen del Accessus y el segundo, fragmentos de las Glossulae, a pesar de que la propia autora reconoce que ninguno de los textos se corresponde con la obra de Arnulfo ${ }^{8}$. La propuesta y las observaciones de Swanson plantean este y otros problemas. En primer lugar, en cuanto al resumen de las Metamorfosis, seguramente habría que decantarse por una opción intermedia, puesto que $L$ y el Accessus del maestro de Orleans comparten las categorías abordadas (materia, intentio auctoris, utilitas y cui parti philosophiae supponatur) pero no ofrecen coincidencias textuales: lo más probable es que ambos se hayan servido de modelos similares, esto es, los varios accessus ovidianos en circulación durante los siglos XI y XII9. Por otro lado, es insostenible la identificación de las notas que siguen al resumen con fragmentos de las Glossulae, dado que no hay correspondencia alguna en los contenidos y, además, $L$ incluye citas de la literatura cristiana, por ejemplo de San Agustín (in psalm. 30.2 serm. 3.7), totalmente ausentes en la obra de Arnulfo. Por todo ello, parece más acertada la brevísima descripción

${ }^{6}$ Mestres, "Un manuscrito", 77.

7 Ibidem.

${ }^{8}$ Swanson, Tradicions, I, 164-6.

9 Véase D. T. Gura, A critical edition and study of Arnulf of Orléans' philological commentary to Ovid's Metamorphoses, Columbus 2010, 58-9 [<rave.ohiolink.edu/etdc/ view?acc_num=osu1274904386>]. 
de Mestres, confirmada desde la primera oración de estas notas (omnia nomina metallorum non abent pluralia). Por último, la identificación del texto escrito por cada mano que postula Swanson tampoco es correcta: la primera transcribe no solo el resumen de las Metamorfosis, sino también la primera línea y media de las notas, lo cual hace pensar que ambos textos iban ya seguidos en el antígrafo de $L$.

El texto de Horacio está copiado a una sola columna de entre 39 y 49 versos por página, excepto en el f. $17^{\mathrm{r}}$, distribuido en dos columnas. El manuscrito comienza directamente con el primer verso de la primera carta, mientras que a partir de la segunda consta al margen el destinatario de cada misiva. Entre los dos libros de las Epístolas hay una línea con el éxplicit del primero, el íncipit del segundo y el título de la siguiente carta (liber primus explicit. incipit secundus. ad cesarem augustum), y al final del segundo libro consta asimismo el éxplicit de la obra (finiunt libri epistularum. finitur liber quem scripsit pugnus et ancer $)^{10}$, debajo del cual el copista transcribió dos aforismos medievales de atribución incierta: diceret aliquis: late possessiones adquirendae sunt y studium est uehemens applicatio animi. Las iniciales de cada poema están generalmente adornadas, a veces con decoraciones de tipo vegetal o geométrico (1.1, 3-6, 10, 12-16, 18-21; 2.1$2)$, a veces con rostros humanos de trazo esquemático (1.7, 11, 17; también en 1.15.26, que en $L$-como en otros manuscritos- marca el comienzo de una nueva epístola). Los versos empiezan con una letra mayúscula un poco separada del resto de la línea.

Por lo que se refiere a la puntuación, cabe señalar que se consignan con bastante sistematicidad los signos de interrogación: se cuentan $86^{11}$, cifra no muy alejada de los 115 presentes, por ejemplo, en la edición de David R. Shackleton Bailey ${ }^{12}$. A pesar de que no puede emprenderse aquí, sería interesante un estudio pormenorizado de los mismos y de su posible contribución a la edición crítica del texto horaciano, puesto que el papel de los códices a este respecto ha sido en buena medida soslayado después de la meritoria edición de Keller-Holder ${ }^{13}$. Un caso ilustrativo se encuentra en 1.6.7 ludicra quid plausus et amici dona Quiritis, en el que la tradición manuscrita se divide entre puntuar tras ludicra, como $L$ (así también los

${ }^{10}$ Así lo lee acertadamente Rubio, Catálogo, 304. La interpretación de la segunda oración no plantea problemas: "Termina el libro que han escrito el puño y la oca", con ancer como grafía medieval en lugar de anser. Swanson, Tradicions, II, 29, sin embargo, da la inexplicable lectura finitur liber quem scripsit pugnes et ancester.

${ }^{11} 1.1 .3,39,44-6,64,69,76,82,90,91,100,102,105 ; 1.2 .23,33,39 ; 1.3 .7,8,9,12,14,20$, 21; $1.4 .5,11 ; 1.5 .12,19,20 ; 1.6 .5,7,8,12,14,29$ (bis), 42; $1.7 .62,63 ; 1.10 .8,18,19,21 ; 1.11 .1-2,3$ (bis), 4-7; 1.15.11; 1.16.10, 14, 17, 31, 75; 1.17.38; 1.18.18, 19, 82, 106; 1.19.14, 20; 1.20.7, 16; 2.1.41, 42, 91 , 206 (bis), 267; 2.2.19, 38, 57, 63 (bis), 80, 86, 90, 148 (bis), 205, 207, 211, 212.

${ }_{12}$ Shackleton Bailey, Opera.

${ }^{13}$ Keller, Holder, Opera. 
propios Keller-Holder o Klingner ${ }^{14}$ ), o después de quid (así Shackleton Bailey y la mayor parte de los editores). Habría que prestar atención, asimismo, a los otros signos de puntuación, lo que puede ejemplificarse con 1.14.43 optat ephippia bos piger optat arare caballus, donde el debate reside en puntuar antes o después de piger (así $L$ ) y, por consiguiente, en hacer concordar el adjetivo con caballus o con bos ${ }^{15}$. Mucho menos sistemáticas son las indicaciones de cambio de párrafo, de las que se registran únicamente 15 ejemplos ${ }^{16}$, aunque por lo general coinciden con las divisiones internas que presentan los poemas en las ediciones modernas.

El códice contiene abundantes glosas tanto marginales como interlineales, sobre todo en las tres primeras páginas, reduciéndose su número a partir del f. $2^{v}$ y volviéndose más irregular, con folios muy anotados y otros más vacíos. Por lo general estas notas son comentarios explicativos o aclaraciones para una mejor comprensión del texto, pero hay igualmente variantes de lectura y correcciones. En ocasiones los versos de Horacio y las glosas parecen ser obra del mismo copista y pueden llegar a confundirse, lo que se intenta evitar mediante el empleo de la interpunción. La mayoría, sin embargo, están escritas por otra mano, muy parecida a la del texto principal y de la misma cronología -como se deduce de la comparación de letras especialmente significativas como la $a$ o la $r$-, distinguibles por su distinto tamaño y por el color de la tinta. Comentario aparte merecen las anotaciones del f. $17^{\mathrm{v}}$, en letra gótica cursiva, que no guardan relación aparente con ninguno de los dos textos de la página y que cabe situar del s. XIII en adelante.

La cubierta del códice reaprovecha parte de un leccionario, fechable por el tipo de letra -una minúscula carolina más redonda- en el s. $\mathrm{XI}^{17}$ : se trata de un bifolio en pergamino con texto a dos columnas, cuyos bordes se han doblado unos dos o tres centímetros hacia el interior del cuaderno. La cara exterior está muy desgastada y oscurecida, mientras que la opuesta se halla perfectamente conservada, lo que contribuye a la identificación del contenido de la guarda. El folio izquierdo porta dos capítulos del Contra Iudaeos, paganos et arianos sermo de symbolo de Quodvultdeus (11-12: uos inquam conuenio - manibus concidencium id est), precedidos por un pasaje de otra obra no identificada, pues la erosión del pergamino apenas si permite identificar alguna palabra suelta. El derecho contiene varios pasajes del Evangelio según Mateo (21.1-2: In illo tempore cum appropinquaret Iesus Hierosolimis et

${ }^{14}$ F. Klingner, Q. Horatius Flaccus. Opera, Berlín-Nueva York 2008³ [1959].

15 Véase H. H. Huxley, "Bos, Bentley, and Byron”, G\&R 19 (2), 1972, 187-9 [<doi. org/10.1017/S0017383500019859>]; B. Baldwin, “Bos Piger or Piger Caballus?”, G\&R 20 (2), 1973, 122-3 [<doi.org/10.1017/S0017383500018611>].

16 1.1.20, 41, 70, 83; 1.7.46; 1.10.22; 2.1.50, 79, 139, 168, 232; 2.2.41, 55, 70, 109.

17 Swanson, Tradicions, II, 28-9. Mestres, "Un manuscrito", 77, habla de "fragmentos de Homilias del siglo IX o X", lo que se antoja una fecha demasiado alta para la paleografía del documento. 
uenisset Bethphage - et adducite michi et reliqua), de Juan Crisóstomo (Homilía 37: Puto res ipsa - ut ea quae sunt destrueret) y del Antiguo Testamento (Isaías 30.27: ecce nomen Domini - eius repleta sunt in-). La sobrecubierta, en pergamino de color claro, es moderna y lleva una hoja de papel encolada en la parte interior.

Por último, en lo que a la cronología se refiere, Mestres fecha el manuscrito entre finales del s. XI y principios del XII; Cordoliani, en el s. XIV; Rubio, en el s. XI, y Castillón -que recoge literalmente la información del inventario del Archivo Capitular de Lérida de $1954^{18}$ y no da muestras de haber hecho una autopsia directa del documento-, en el s. XII ${ }^{19}$. Swanson sitúa su copia a finales del s. XII por la atribución del texto del f. $17^{\mathrm{v}}$ a la obra de Arnulfo de Orleans ${ }^{20}$, pero dicha identificación, como se ha indicado, es insostenible. Así pues, y teniendo en cuenta la angulosidad de la carolina empleada, parece más prudente considerar una datación genérica en el s. XII.

\section{Historia DEL Códice}

Como puede colegirse de su denominación, el códice perteneció en origen a la colección de la catedral de Roda de Isábena (Huesca), y con toda probabilidad fue también allí donde se copió, dado que sus características paleográficas permiten compararlo con otras producciones contemporáneas del escritorio ribagorzano ${ }^{21}$. Para disponer de una primera mención del documento hay que esperar hasta el viaje a Roda que hizo Jaime Villanueva en 1806, en cuya narración nos habla de la existencia en el archivo de "fragmentos abundantes de las epístolas de Horacio, ms. del siglo XII, y otros ítem de Homero; breve comentario incógnito de algunas comedias de Terencio, ms. en el siglo XIII"22. A raíz del concordato de 1851 entre la Santa Sede y España, la colegiata de Roda fue suprimida, y en 1863 se ordenó por Real Decreto el traslado de sus volúmenes a la catedral de Lérida ${ }^{23}$. El inventario de los documentos, elaborado por Mateo de Lasala al año siguiente, recoge la siguiente entrada para el códice nº 7: "Fragmentos de las epístolas de Horacio, manuscrito del siglo 12, algunos de Homero; comentarios de algunas comedias de Terencio. Consta de 17 fojas con cubiertas de pergamino"24. No

18 ACL, P0_M1_P3_C07_CM05: Inventario de los códices y libros impresos que pertenecen al archivo y biblioteca de la S. I. Catedral de Lérida y están depositados en el seminario.

19 Mestres, "Un manuscrito", 77; Cordoliani, "Inventaire”, 378; Rubio, Catálogo, 303; Castillón, "Catálogo", 137.

${ }^{20}$ Swanson, Tradicions, II, 31.

${ }^{21}$ Swanson, Tradicions, II, 28.

${ }^{22} \mathrm{~J}$. Villanueva, Viage literario a las iglesias de España, vol. XV: Viage á Gerona y á Roda, Madrid 1851, 171.

${ }^{23}$ Véase Castillón, "Catálogo", 134-7.

${ }^{24}$ ACL, P0_M1_P3_C07_CM01: Inventario de los códices, diplomas y otros documentos 
hay duda, por la descripción y, en concreto, por el número de folios, de que se trata de $L$, pero Lasala se habría basado en las notas de Villanueva (si no en otra fuente o catálogo), sin comprobar el contenido del manuscrito, y no se habría dado cuenta de que los cuadernos correspondientes a la Ilias Latina y a Terencio se hallaban ya perdidos: lo único que se conservaba eran las epístolas horacianas ${ }^{25}$. El mencionado inventario de 1954, aunque posterior al artículo de Mestres de 1949, arrastra los errores de Lasala ${ }^{26}$ :

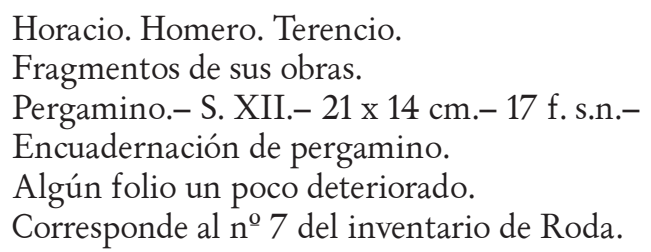

Es imposible no aplaudir la capacidad predictiva del eterno Horacio, pues no deja de ser una ironía del destino que un manuscrito con la célebre Epistola 1.20, en la que el poeta predice a su libro que uinctus mitteris Ilerdam (1.20.13), haya terminado final y efectivamente en Ilerda, topónimo que nuestro códice se encarga de glosar con cierta gracia: ciuitatem ubi mala carmina distribuebant ad species mittendas (f. $12^{\mathrm{r}}$ ).

\section{Colación de las EPÍSTOLAS de HoRAcio}

La presente colación parte del texto de la cuarta edición teubneriana de Shackleton Bailey ${ }^{27}$. Primero ofrezco la lectura de este editor y, separada por dos puntos, la de $L$. Se incluyen todas las lecturas ante correctionem $\left(L^{a c}\right)$ que han podido ser determinadas, a pesar de que en muchos casos se trata, con toda probabilidad, de errores de copia subsanados al momento por el propio amanuense. $L^{c}$ indica estas correcciones del copista y las que se han llevado a cabo mediante rasura. Aquellas realizadas por alguna de las manos que transcriben las glosas, en cambio, se recogen bajo la sigla colectiva $L^{2}$, pues todas ellas ofrecen textos en una minúscula carolina angulosa datable, como la mano principal, en el s. XII, lo que impide distinguirlas con facilidad.

pertenecientes al archivo de la ex-catedral de Roda, formado por D. Mateo de Lasala $y$ Villanova, doctor en derecho y Ayudante de $2^{\circ}$ grado del cuerpo facultativo de Archiveros-Bibliotecarios, comisionado al efecto por Real orden de 21 de Julio de 1864; cuyos documentos han sido trasladados y depositados en el archivo de la Catedral de Lérida por Real orden de 20 de Diciembre de 1863. Habiendo sido comisionado por el Ilmo. Sor. obispo de Lérida para la traslación y recibo de dicho archivo el I. S. D. D. Isidro Valls y Pascual, Dignidad de Arcipestre (sic) de la misma Sta. Iglesia, f. $2^{\mathrm{v}}$, $\mathrm{n}^{\circ} 7$.

${ }^{25}$ Mestres, "Un manuscrito", 76-77.

26 ACL, P0_M1_P3_C07_CM05: Inventario, $\mathrm{n}^{\mathrm{0}} 2$.

27 Shackleton Bailey, Opera. 
He marcado con un asterisco las lecturas aparentemente exclusivas de $L$, una vez examinados el reducido aparato crítico de Shackleton Bailey y los mucho más nutridos de Keller-Holder y Navarro Antolín, amén de las lecturas del Dertusensis $100(T)$ reportadas por Ribas y de los Parisini lat. $7977\left(P_{6}\right)$ y $8213\left(P_{8}\right)$, que he revisado sobre la copia digital disponible en Gallica dada su estrecha relación con $L(\text { infra, } \S 4)^{28}$. Téngase en cuenta, no obstante, el carácter imperfecto y provisional de este marcaje, debido tanto a las fuentes empleadas como a una serie de motivos que se expondrán a continuación.

\section{LIBER I}

1. 6 rediens : tociens | exoret : exornet 7 purgatam crebro qui : *qui crebo purgatam $\bullet 12$ possim : "possum $L^{a c} \bullet 14$ addictus : adductus $L^{a c} \bullet$ 31 cheragra : ciragra $\bullet 32$ quadam : quodam $\bullet 45$ mercator ad Indos : *ad Indos mercator $\bullet 52$ est auro : *inu. ord. $\bullet 57-58$ inu. ord. $\bullet 57$ est lingua : et $1-\bullet 58$ sed : si $L^{c} \mid$ desunt : desint $\bullet 65$ qui : *quae $L^{c} \bullet 76$ es<t> : es $\bullet 78$ frustis : *frustris $\bullet 82$ horam : hora $\bullet 87$ fabri : *o fabri • *97 om. $L^{1}$ (in marg. add. $\left.L^{2}\right) \cdot 98$ spernit : *spernat $L^{a c}$

2. ad lollium maximum in marg. $\bullet 4$ planius : plenius $\bullet 8$ aestus : (a)estum $\bullet 10$ quid : quod $\cdot 23$ Circae : circes $\bullet 25$ fuisset turpis : ${ }^{*}$ inu. ord. $\cdot 31$ cessantem : cessatum | somnum : curam $\cdot 32$ hominem : homines $\cdot 33$ atqui : atquin $\cdot 34$ curres : cures $\cdot 38$ oculum: occulos | quid : quod $\cdot 51$ aut : ac $\cdot 52$ fulmenta : fomenta | podagrum : podagram $\bullet 54$ acescit : *accessit $L^{a c}:$ acessit $L^{c} \bullet 65$ qua : quam | monstret : $\operatorname{mo}(\mathrm{n})$ strat $\cdot 67$ abbibe $:$ ad(h)ibe $\bullet 68$ uera : uerba

3. ad iulium florum in marg. 3 Hebrusque : *ebrus -8 diffundit : diffudit $\cdot 20$ furtiuis : *furtiuus $L^{a c} \cdot 22$ et : *et nec $\cdot 29$ uolumus : cupimus $\bullet 30$ <est> om. $\bullet 32$ nequiquam : necquicquam | ac : at $\bullet 33$ seu... seu : heu... heu | ueri : rerum

4. ad albium elegorgium in marg. $\bullet 7$ dederunt : *dederint $\bullet 9$ qui : quam $\bullet 11$ mundus : modus et $\bullet 12$ timores : *timorem

5. ad torquatum in marg. 12 fortunam : fortuna 15 uel : *uelut $L^{1}$ - 16 dissignat : designat | operta : *aperta $L^{1} \cdot 17$ inertem : inermem • 20 quem : *et quem $\cdot 23$ ne non : nec non $\cdot 25$ ut : et $\cdot 26$ Septiciumque : septimiumque | *adhibe ad finem $u$. interpol. 27 cena prior : *cena $L^{1}$ : *prior cena $L^{2} \cdot 28$ assumam : ad summam

6. ad numicium in marg. 10 utrubique : utrobique 11 exsternat : exterret $\bullet 16$ ipsam : ${ }^{*}$ ipse $\bullet 20-23$ post 48 transt. : inter 19 et 24 praeb. - 22 qui : quod $\cdot 26$ uia : et uia $\cdot 27$ quo : *qua $\cdot 28$ aut : et $\cdot 30$ una : *una est $L^{a c} \cdot 31$ et : ut $\bullet 34$ et $\mathrm{om} \cdot \bullet 35$ quadret : quadrat $\bullet 40$ chlamydes Lucullus : *lucullus clamides $\bullet 48$ primus : primum $\bullet 50$ laeuum : s(a)euum - 51 fodicet : fodiat $\bullet 53$ fascis : *faces $\bullet 55$ facetus : *factus $L^{1} \bullet 56$ lucet :

${ }^{28}$ Shackleton Bailey, Opera; Keller, Holder, Opera; Navarro Antolín, Epístolas; Ribas, Riber, Sàtires. Parisinus lat. 7977: <https://gallica.bnf.fr/ark:/12148/btv1b9068281k> [17/07/2019]. Parisinus lat. 8213: <https://gallica.bnf.fr/ark:/12148/btv1b10035826v> [17/07/2019]. 
licet $\bullet 57$ ducit : ducet $\bullet 59$ Campumque : populumque $\bullet 60$ *ut om. 65 Mimnermus : *numerinus in marg. $L^{2} \cdot 68$ nil : non

7. ad mecenatem in marg. 6 dissignatorem : dessignatorem | lictoribus : *littoribus $L^{1} \bullet 10$ si bruma : *inu. ord. $L^{2} \mid$ illinet : *illinent s. $l$. $L^{2} \cdot 13$ concedes : concedas | hirundine : *hyrunde $\cdot 20$ stultus : *tultus $\bullet 22$ ait : agit | paratus : paratum $\cdot 26$ angusta : *in a- $L^{2} \cdot 29$ cornicula : uulpecula 39 inspice : *inspicie $L^{a c} \bullet 41-42 * i n u$. ord. (corr. manus recentior) $\bullet 46$ causisque : ${ }^{*}$ causis $\bullet 50$ arrasum : abrasum $\bullet 51$ resecantem : purgantem $\bullet 53$ *sit post unde $s c r . \cdot 55$ Vulteium : uulteum - 56 censu : *sensu $\bullet 63$ neget : negat $\bullet 64$ neglegit : negligit | Vulteium : uulteum $\bullet 71$ uenies : *uenias | *rem om. $L^{1} \cdot 73$ dormitum : *dormit $L^{1}$ | dimittitur om. et ad finem $u$. add. | hinc : hic $L^{c}$ : *hic est $L^{a c} \bullet 83$ est om. $\bullet 89$ aedis ((a)edes ad finem $u$. scr.) : *artes $\bullet 96$ semel : simul

8. ad celsum albinouanum in marg. -3 quaeret : qu(a)erit $\cdot 5$ oleamue : oleamque

9. ad claudium neronem in marg. $\bullet 1$ intellegit : intelligit

10. ad aristium fuscum in marg. 3 at : ad $\cdot 9$ ad : in $\mid$ fertis : effertis 18 diuellat : depellat $\cdot 20$ rumpere : ${ }^{*}$ rumperem ut uid. $L^{\text {ac }}$ • 23 laudaturque : *laudatur quia $\bullet 25$ fastidia : fastigia $\bullet 27$ Aquinatem : *aquinantem $L^{a c} \cdot 28$ medullis : *medullas $L^{a c} \bullet 31$ quatient : *quassient $L^{1} \cdot 33$ praecurrere : * praecurre $\bullet 40$ uehit : uehet $\bullet 41$ seruiet : *seruet $L^{1}$ | nesciet : *nescient $L^{a c}$

11. ad bulacium in marg. $\cdot 3$ minorane : minoraue $\cdot 8$ uicus : *uicis $L^{1} \bullet 15$ si te : inu. ord. $\bullet 17$ Mytilene : *mictiline | pulchra facit quod : *facit quod pulcra $\bullet 18$ auris : austris 19 Sextili : *sextilis $L^{a c} \bullet 20$ ac : et $\cdot 23$ neu : nec

12. ad iccium in marg. 5 uentri bene : *inu. ord. $\bullet 7$ sin : si $\bullet 9$ riuus : *riuis $\bullet 12$ Democriti : *democti $L^{1}:$ *democri $L^{2} \bullet 13$ *est om. $\bullet 21$ seu porrum : *et p- 23 nil : nec $\bullet 27$ Phraates : *pharatres $\bullet 29$ defudit : diffundit

13. ad uinnium asellium in marg. $\bullet 6$ chartae : *quart(a)e $\bullet 12$ seruabis : *serutabis $L^{a c} \cdot 14$ glomus : glomos $\bullet 16$ neu : nec $\bullet 17$ possint : possunt - 19 uade : uive

14. ad uillicum suum in marg. 9 auet : amat 18 bis scr. 19 nam quae : namque $\cdot 28$ disiunctum : *disiunctu $L^{c} \cdot 31$ quid nostrum concentum : concentum quid nostrum $\bullet 32$ nitidique : nitideque $L^{a c} \bullet$ 33 scis : *nescis $\bullet 40$ rodere : *reddere $\bullet 44$ scit : *sit | censebo exerceat artem : *artem censebo excerceat

15. ad numonium ualam in marg. $\bullet 5$ relinqui : *reliqui $\bullet 10$ deuersoria : diuersoria $\bullet 12$ *Baias om. $\bullet 15$ bibant : *bibes $L^{a c} \bullet 16$ iugis : dulcis $\bullet$ 17 perferre : sufferre $\cdot 26$ noua epist. inc.; ad menium in marg. $\bullet 28$ certum : *ciuem $L^{a c} \cdot 29$ dinosceret : *disnoceret

16. ad quincium in marg. -3 an pratis : et p- $\mid$ amicta : amica $\bullet$ 4 *forma om. | situs : *scitus $\cdot 7$ decedens : discedens -8 benigni : benign(a)e $\bullet 14$ utilis, utilis : aptus utilis $\bullet 23$ dissimules : *dissimiles $\bullet$ 26 permulceat : demulceat $\cdot 27$ an : *aut $\bullet 28$ et tibi : *tibi $\bullet 32$ prudens : sapiens $\bullet 38$ falsis : *falcis $\bullet 40$ medicandum : mendicandum $\bullet 43$ res sponsore : responsore $\bullet 45$ speciosum $:{ }^{*}$ et speciosum $\bullet 46$ nec furtum : *hoc furtum (fortum $L^{1}$ ) $\mid$ dicit : dicat $\bullet 49$ negitatque : negat atque $\bullet 51$ 
suspectos : suspectus $L^{a c} \mid$ et : ad $\bullet 56$ *tuum ante mihi scr. $\bullet 59$ clare $^{2}$ om. $\bullet 61$ iusto sanctoque : sanctum iustumque $\bullet 62$ *faudibus om. $\bullet 72$ penusque : penumque $\bullet 73$ sapiens : prudens $\bullet 75$ *bona $\mathrm{om}$.

17. ad sceuam in marg. -2 tenuem : tandem $\mid$ deceat : liceat $\bullet 11-$ $12 \mathrm{om}$. et in marg. add. 17 potior : *pociore $L^{a c} \cdot 21$ poscis : *possis | rerum : uerum es $\cdot 28$ quidlibet : *quoslibet $\cdot 30$ angui : angue $\cdot 31$ chlanidem : clamidem $\cdot 32$ ineptus : *inemptus $\cdot 36$ non : nec $\cdot 38$ fecitque : fecitne | atqui : atquin $\bullet 39$ aut : *aud $\bullet 42$ aut : *aud $\bullet 43$ sua : suo $\bullet 46$ paupercula : *et paupercula $\bullet 52$ Brundisium : *brandusium

18. ad lollium in marg. 1 prior post si scr. et exp. $\bullet 5$ prope : et prope $\bullet 8$ om. $L^{1}$ (in marg. add. $\left.L^{2}\right) \cdot 14$ mimum : nimium $\bullet 15<$ et> om. - 20 Brundisium : *brandusium | Minuci : numici | uia om. et ad finem u. scr. $\cdot 22$ unguit : ungit $\cdot 29$ patiuntur : paciantur $\cdot 31$ Eutrapelus : *eutrapeus $\bullet 35$ pascet : pascit | ad : *ac $\bullet 37$ illius : ullius $\bullet 39$ reprendes : reprehendes $\bullet 45$ educet : educit | agros : *agro 46 Aeoliis : (a)etholiis - 51 canem : *canes $\bullet 52$ *adde om. $L^{1} \bullet 57$ aruis : armis $\bullet 63$ *tuus post frater scr. | "est post Hadria scr. $\bullet 74$ pueri dominus : inu. ord. $\bullet 77$ incutiant : *incuciunt $\bullet 78$ *quondam om. $L^{1}:{ }^{*}$ aliquando s. $l . L^{2} \cdot 82$ Theonino : *theonico | ecquid : *hecce quid $\bullet 87$ metuet : metuit $\bullet 91$ nocte (uel nocte ad finem $u$. scr.) : luce $\bullet 94$ supercilio : *supercilium $L^{1} \bullet$ 98-99 ne... neu : non... non 107 et : ut | ${ }^{*}$ mihi $^{2}$ om. $\cdot 110$ dubiae : *dubite $L^{a c} \bullet 111$ sed : *nec| *est om. | quae : qui | ponit : donat $112 \mathrm{mi}$ om. et ad finem u. scr.

19. ad mecenatem in marg. $\bullet 5$ fere : *feres $L^{a c} \bullet 8$ prosiluit : prosiliit - 9 seueris : *seueras $L^{a c} \bullet 10$ edixi : edixit $\bullet 12$ si quis uultu toruo ferus : *ferus si quis uultu toruo $\bullet 13$ textore : *textorem $\bullet 20$ uestri : *iuri $L^{a c} \bullet$ 27 modos : *modis $L^{a c} \bullet 30$ alceus post socerum scr. et del. $\bullet 32$ quoque : *et ego | Latinus : latinis • *37-38 om. $L^{1}$ (in marg. add. $\left.L^{2}\right) \bullet 41$ illae : *illinc|spissis : "spul'is (spiritualis?) $L^{a c}$ : *spicis $L^{c} \bullet 44$ manare poetica mella : " poetica mella manare 46 ne secer : *nececer $L^{a c}$ : ${ }^{*}$ necesser $L^{c}$ | ungui : ungue $L^{c} \cdot 47$ iste : ille

20. ad librum suum in marg. 2 Sosiorum : *sociorum $L^{1} \mid$ pumice $:$ " punice $\mid$ mundus (uel mundus ad finem $u$. scr.) : nudus $\bullet 4$ gemis : *genus $\bullet 7$ quid $^{2}$ : quis $\bullet 11$ uel post ubi scr. et exp. 12 aut : *aut (s. l.) et | tineas : "timeas $L^{a c} \bullet 28$ Lepidum : *tepidum $L^{1} \mid$ dixit : duxit $\bullet$ liber primus explicit. incipit secundus

\section{LIBER II}

1. ad cesarem augustum $s . l \cdot \cdot 2$ tuteris : *et tuearis $\bullet 4$ longo : *multo - 16 numen : nomen 18 hoc : hic 19 nostris ducibus : *inu. ord. • 26 annosa : *et annosa $\cdot 28$ Graiorum : graecorum $\cdot 31$ olea : oleam - 33 doctius : *doc $L^{1} \cdot 34$ *pro tempore post dies scr. $\bullet 39$ perficit : prospicit $\bullet 41$ probosque : poetas $\bullet 42$ respuat : respuet $\bullet 45$ permisso : *promisso 46 uello : *ullo $L^{a c} \mid$ *annos post uello scr. | demo unum om. $• 53$ mentibus haeret (in marg. $L^{2}$ ) : *mente tenetur $\bullet 56$ Pacuuius : *passemius • 58 Epicharmi : *epycomedias $L^{1} \bullet 60$ ediscit : edicit • 66 quaedam : *quadam $\bullet 69$ delendaue : delendaque $\bullet 70$ memini quae : meminique $\bullet 73$ quae : ${ }^{*}$ qu(a)eque $\bullet 77$ illepideue : inlepideque $\bullet 83$ 
ducunt : dicunt $\bullet 105$ rectis : certis 108 mentem populus : ${ }^{*}$ inu. ord. $\bullet$ $113{ }^{*}$ et $^{1}$ om $\cdot \bullet 114$ habrotonum : abrotanum | aegro : *ego $L^{1} \bullet 123$ siliquis : *si aliquis $L^{a c} \cdot 126$ *balbum om. $L^{1}$ (s. l. add. $\left.L^{2}\right) \cdot 140$ leuantes : leuiates ut uid. $\bullet 142$ pueris : et p- 145 inuecta : inuenta 149 coepit uerti : inu. ord. $\bullet 163$ Thespis : *respis | Aeschylus : (a)eschinus • 167 inscite : in scriptis $\bullet 168$ arcessit : accessit 173 Dossennus : *dorcennius - 175 demittere : dimittere $\bullet 176$ stet : *stat 177 scaenam : *senam $\bullet 182$ etiam : *et I poetam : *pociam $\bullet 184$ depugnare : *pugnare $\bullet 190$ cateruae : *ateue 193 captiua : *et c- 196 elephans : elephas | conuerteret : *conueteret 198 sibi : *tibi $L^{1} \mid$ nimio : *mimmo 205 scaena : *cena - 213 et magus ut : ut magus et -220 uineta : *uinaeta $L^{a c} \mid$ caedam : *si c- 221 unum : *uinum $L^{a c} \cdot 223$ reuoluimus : reuolumus $L^{1} \bullet$ 226 speramus : *superamus $L^{1} \mid$ eo rem uenturam : *item uenturum $L^{1}$ : item fore uenturum $L^{2} \cdot 228$ arcessas : *accerces $\bullet 234$ nomisma : *nummisma $\bullet 235$ remittunt : *remitatur $L^{1} \bullet 236$ foedo : *ferso $L^{1} \bullet 239$ uetuit : "posuit $L^{1} \bullet 240$ aut : *ut 243 haec : *hoc $L^{1} \bullet 244$ Boeotum : boetum $\bullet 245$ neque : *namque $L^{a c} \cdot 246$ munera quae multa : *multa qu(a)e munera ${ }^{*}$ qu(a)e post dantis scr. $L^{1}\left(\right.$ del. $\left.L^{2}\right) \cdot 248$ *hos post uultus scr. $L^{1}$ (del. $\left.L^{2}\right) \cdot 252$ arces : *artes $\bullet 258$ maiestas : *magestas • 261 *se om. $\cdot 264$ ficto : *fixo $L^{1} \cdot 265$ usquam : umquam $\bullet 267$ ne : nec - 269 tus : et tus

2. ad iulium florum in marg. -2 si quis forte : *forte (om. et in marg. add.) si quis $\bullet 3$ hic et : *et hic $\bullet 8$ quiduis : "quosuis $L^{a c}$ : quiuis $L^{c}$ | imitaberis : imitabitur $\bullet 14$ quiuis : *quis $\bullet 16$ nihil te si : *si te nichil | laedit : *ligat 1(a)edat $\bullet 23$ quid : *qui $\bullet 24$ quereris : *queres $\bullet 39$ catus : *cautus $\bullet 44$ uellem : possim $\bullet 52$ uersus facerem : ${ }^{*}$ inu. ord. | habentem : *hauertem $\bullet 53$ expurgare : expugnare $\bullet 66$ curas : *curam $\bullet 70$ haud sane : humane $\bullet 71$ purae : plures $\bullet 75$ fugit canis : *inu. ord. $\bullet 77$ urbem : urbes - 80 contracta : contacta $\bullet 83$ om. et in marg. add. | curis : *canis $L^{a c}$ - 89 huic... ille : hic... illi 101 Mimnermus : *mimeruus 112 fruentur : ferentur 128 haud : haut 130 uacuo : *uacuos $L^{a c} \cdot 134$ insanire : *insaniare $L^{a c} \bullet 139$ extorta : *exorta 140 om. et in calce add. 145 recordor : *recordabor $L^{1} \bullet 147$ quod : quid $\bullet 149$ herba : *herbis $\bullet 151$ proficiente : *proficientem $\bullet 156$ timidumque : *timidum $\bullet 158$ mercatus et aere est : mercatur et h(a)ere $L^{1}:$ mercatus et h(a)ere $L^{2} \bullet 161$ daturas : daturus $\bullet 164$ fortasse : *fortassis $L^{a c} \bullet 167$ Veientis : uegentis $\bullet 171$ refringit : refugit $\bullet 174$ permutet : ${ }^{*}$ permutte $\bullet 175$ sic : sed $\bullet 176$ alternis : alterius - 180 *ebur om. $\bullet 197$ ac potius puer ut : *an puer ut potus $\bullet 199$ domus procul : inu. ord. $\bullet 200$ an : *aut $\bullet 201$ tumidis uelis : *tumidisue $\bullet 204$ extremi : *etremi $\bullet 211$ fis : sis ut uid. $\bullet 212$ leuat : iuuat $\bullet 213$ recte nescis : inu. ord. | peritis : *a p- $\bullet 215$ ne : *nete 216 decentius : licencius ${ }^{29} \bullet$ finiunt libri epistularum. finitur liber quem scripsit pugnus et ancer

\section{RELACIÓN DE $L$ CON OTROS MANUSCRITOS HORACIANOS}

Antes de intentar trazar algunas conclusiones a partir del análisis de las lecturas de $L$, es menester advertir que la cifra de manuscritos conservados de

${ }^{29}$ Swanson, Tradicions, II, 29 lee hecencius. 
Horacio asciende a varios centenares y que, de ellos, solo un reducido número ha sido estudiado, de manera que, por ahora, no parece posible ni establecer el estema de la transmisión del texto ni resolver muchos de los problemas que esta plantea ${ }^{30}$. A dicho escollo inicial se suma el hecho de que el antígrafo o un ancestro de $L$ contenía sin duda variantes de lectura marginales o interlineales, lo cual dificulta aún más su clasificación. El argumento más destacado para aseverarlo lo constituyen tres lectiones singulares del rotense que se derivan de la interpolación de esas variantes dentro del texto principal: esto es, encontramos dos palabras cuando en el verso cabe solo una, generando secuencias amétricas, y cada una de estas palabras se atestigua en otros códices y procede, por tanto, de ramas diferentes de la tradición. Se trata de los pasajes 1.3.22 et nec, 1.7.73 hic est (con est suprimido mediante rasura, seguramente por $L^{2}$ ) y 2.2.16 ligat l(a)edat. A ellos habría que añadir otros lugares como 1.7.89 artes, 1.18.91 luce y 1.20.2 nudus, donde al final de las respectivas líneas la mano principal ha escrito (a)edes, uel nocte y uel mundus -el texto comúnmente editado- sin llamada alguna al verso, lo cual parecería indicar que estas lecturas no son producto de una colación sino que su fuente directa es un ancestro de $L$.

Si bien no es posible plantear la inclusión del rotense en una familia de manuscritos, puesto que todos los intentos de agrupación han resultado poco satisfactorios o han recibido numerosas críticas (por los títulos de las cartas, por ejemplo, $L$ podría incluirse dentro de la clase $\Psi$ de Klingner $^{31}$, pero considérese que para la mayor parte de las Epístolas la otra rama, $\Xi$, está representada por un único testimonio, $C$ ), sí deben señalarse aquellos códices con los que mantiene una relación más estrecha. Para mayor comodidad del lector, doy a continuación las siglas empleadas ${ }^{32}$ (véase página siguiente).

Mestres hizo una primera aproximación a la cuestión y, entre otras afirmaciones mucho más discutibles, acertó a ver numerosos errores conjuntivos con $T$, datable posiblemente en el s. XI y de origen incierto ${ }^{33}$. Una cifra aún mayor de correspondencias la ofrece $L$ con los Parisini ya mencionados, $P_{6}$ y $P_{8}$. Estos cuatro manuscritos coinciden en 1.13 .19 uiue, 1.15.17 sufferre, 1.16.61 sanctum iustumque y 1.17.38 fecitne y, además, comparten ad lollium maximum como título para 1.2; se avanzan así más

${ }^{30}$ Véase G. Pasquali, Storia della tradizione e critica del testo, Florencia 1952², 374-385; R. J. Tarrant, "Horace", en L. D. Reynolds (ed.), Texts and Transmission: A Survey of the Latin Classics, Oxford 1983, 182-6.

${ }^{31}$ Klingner, Opera.

${ }^{32}$ Me baso principalmente en el conspectus siglorum de la edición de Navarro Antolín, Epistolas, la más completa en ese sentido, aunque añado entre paréntesis, siempre que resulta posible, la sigla utilizada por Keller, Holder, Opera.

33 Mestres, "Un manuscrito", 80-81. Sobre este códice véase H. Denifle, É. Chatelain, Inventarium codicum manuscriptorum capituli Dertusensis, París 1896, 21, n 100 ; Ribas, Riber, Sàtires, xiii-xiv; B. Munk Olsen, L'étude des auteurs classiques latins aux XI et XII siècles, París 1982, I, 496; Rubio, Catálogo, 548-9, nº 672. 


\begin{tabular}{|c|c|}
\hline Sigla & Manuscrito \\
\hline$B_{2}$ & Londiniensis Harleianus 2724, saec. XI \\
\hline$B_{5}$ & Londiniensis Regius 15 B VII, saec. XII \\
\hline$C($ Keller: $E)$ & Monacensis lat. 14685 , saec. XI \\
\hline$E($ Keller: $\varepsilon)$ & Einsiedlensis 361 , saec. $X$ \\
\hline flor. Nostr. & Florilegium Nostradamense, saec. XII \\
\hline$G$ (Keller: $g$ ) & Gothanus B 61, saec. XV \\
\hline$M($ Keller $)$ & Mellicensis 1545 , saec. XI \\
\hline$P_{3}($ Keller: $\lambda)$ & Parisinus lat. 7972 , saec. $X$ \\
\hline$P_{5}($ Keller: $\gamma)$ & Parisinus lat. 7975 , saec. XI \\
\hline$P_{6}$ & Parisinus lat. 7977 , saec. XI \\
\hline$P_{7}$ & Parisinus lat. 7978 , saec. XI-XII \\
\hline$P_{8}($ Keller: $v)$ & Parisinus lat. 8213 , saec. XI-XII \\
\hline$P_{11}($ Keller: $\pi)$ & Parisinus lat. 10310 , saec. $I X-X$ \\
\hline$P_{12}$ & Parisinus lat. 16689, saec. XI \\
\hline$T$ & Dertusensis 100 , saec. XI \\
\hline$V_{3}$ & Vaticanus Reg. lat. 1666, saec. XI \\
\hline$V_{g}$ & Vaticanus lat. 3258, saec. XI \\
\hline$V_{12}$ & Vaticanus lat. 1657, saec. XII-XIII \\
\hline
\end{tabular}

de medio milenio a Meineke, a quien los editores suelen atribuir el haber interpretado maxime (1.2.1) como vocativo del cognomen y no del adjetivo. Los dos parisinos y el rotense presentan todavía otra coincidencia, 1.16.26 demulceat, y para un error en 1.18 .111 puede trazarse un mismo origen: mientras que $L$ ofrece nec, $P_{6}$ y $P_{8}$ leen, junto con $V_{9}, h(a) e c$. Hacia una filiación común de los tres, incluyendo tal vez el dertosense, apuntaría el hecho de que distintas glosas o variantes interlineales de los códices parisinos permiten explicar algunas lectiones singulares de $L$ : por lo que se refiere a $P_{8}$, uel nec (f. $44^{\mathrm{r}}$ ) para 1.3.22 et nec, de qua terra sit (f. $47^{\mathrm{r}}$ ) para 1.7.53 unde sit, et (f. $\left.52^{\mathrm{v}}\right)$ para 1.16 .45 et speciosum y et $\left(\mathrm{f} .57^{\mathrm{v}}\right)$ para 2.1.26 et annosa; a su vez, la más ilustrativa de $P_{6}$ es processus temporis (f. $84^{\mathrm{r}}$ ) para 2.1.34 dies pro tempore. En dos pasajes, por último, se da la situación de que $L$ comparte lectura con $P_{6}$ y con una manus correctrix de $P_{8}: 1.17 .36$ nec y 1.19.8 prosiliit.

Antes de terminar con esta somera valoración de las lecturas de $L$, conviene reseñar aquellos lugares en los que coincide de manera exclusiva con un solo manuscrito:

- 1.7.6 dessignatorem, 2.1.70 meminique y 2.1.223 reuolumus con $P_{5}$;

- 1.12.23 nec y 2.1.114 abrotanum con $P_{8}$; 
- 1.6.56 licet y 1.18.5 et prope con $P_{12}$;

- 2.2.8 quiuis con $E$;

- 1.7.64 uulteum con el flor. Nostr.;

- 1.11.18 austris con $B_{5}$;

- 2.2.53 expugnare con $M$;

- 2.1.60 edicit con $P_{3}$;

- 1.18.35 pascit con $V_{3}, \mathrm{y}$

- 1.16.14 aptus utilis con $V_{g}$.

Dado que no son muy numerosas, consigno también las coincidencias exclusivas con dos códices:

- 1.7.64 negligit con el flor. Nostr. y $P_{8}$;

- 1.16.32 sapiens con $B_{2}$ y $P_{7}$;

- 1.16.73 prudens con $P_{7}$ y $V_{12}$;

- 1.18.8 om. con $G$ y $P_{11}$, y

- 2.1.267 nec con $B_{5}$ y $C$.

Por lo general las variantes no son demasiado significativas, y podrían ser debidas en no pocos casos a un fenómeno de poligénesis. La más destacable es la omisión de 1.18.8, pero ninguno de los dos códices en los que también falta ese verso guarda con $L$ una especial afinidad.

\section{A MODO DE CONCLUSIÓN}

No es mi intención intentar convencer al lector de las bondades del texto de Horacio transmitido por nuestro manuscrito: la realidad es que se trata de un ejemplar con numerosas interpolaciones de notas marginales o interlineales, errores de copia, omisiones de palabras e incluso de versos enteros, transposiciones a menudo amétricas, entre otros problemas. Algunos han sido subsanados por las sucesivas manos correctoras, pero muchos han permanecido en el pergamino y han dado lugar a un texto a todas luces mejorable. De todos modos, no cabe duda de que el estudio sistemático y concienzudo de la miríada de códices horacianos, no solo por lo que se refiere a los poemas sino también a glosas y escolios, ha de conducir, lenta pero firmemente, a un mejor conocimiento de la transmisión de la obra del venusino durante la Edad Media, a una mejor definición de los problemas textuales que la rodean, y a una mejor comprensión de las relaciones entre los distintos scriptoria y bibliotecas del medievo. Sean estas páginas un paso más en el largo camino todavía por recorrer. 